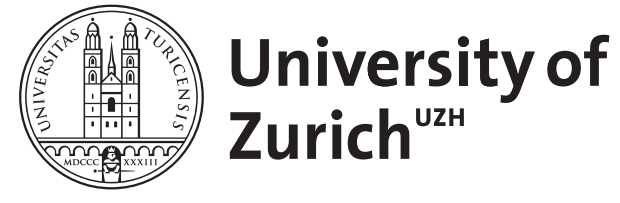

\title{
Can spin-polarized photoemission measure spin properties in condensed
} matter?

Osterwalder, Jürg

DOI: https://doi.org/10.1088/0953-8984/24/17/171001

Posted at the Zurich Open Repository and Archive, University of Zurich ZORA URL: https://doi.org/10.5167/uzh-75103

Journal Article

Originally published at:

Osterwalder, Jürg (2012). Can spin-polarized photoemission measure spin properties in condensed matter? Journal of Physics: Condensed Matter, 24(17):171001.

DOI: https://doi.org/10.1088/0953-8984/24/17/171001 


\title{
Can spin-polarized photoemission measure spin properties in condensed matter?
}

\author{
Jürg Osterwalder \\ Physik-Institut, Universität Zürich, Winterthurerstr. 190, 8057 Zürich, Switzerland \\ E-mail: osterwal@physik.uzh.ch
}

\begin{abstract}
Photoemitted electrons move in vacuum; their quantum state can be completely characterized in terms of energy, momentum and spin polarization by spin-polarized photoemission experiments. A review article in this issue by Heinzmann and Dil [1] addresses the question to what degree the measured spin properties, i.e. the magnitude and direction of the spin polarization vector, can be traced back to the quantum state from which these electrons originate. The answer is a careful 'yes', which is highly relevant in view of the current interest in these experiments and their application to topological insulators, where the spin-orbit interaction produces spin polarized surface states .
\end{abstract}

Photoelectron spectroscopy has been a major probe for the electronic energy levels in atomic, molecular, surface and solid state physics for decades. Based on Einstein's introduction of light quanta of well defined energy and his explanation of the photoeffect [2], the photoelectron spectrum reflects directly the distribution of binding energies in the system, including subtle manybody effects [3]. In angleresolved photoemission (ARPES) from crystalline solids, the periodicity of the lattice makes the electron momenta in the solid a measurable quantity, because at least the component parallel to the surface is conserved and thus reflected in the momentum of the photoelectron. The Topical Review by Heinzmann and Dil in this issue [1] addresses the question whether the same is true also for the electron spin, i.e. whether the measured spin polarization of photoelectrons can be directly related to the spin properties of their quantum state in the condensed matter system. The answer is no, in principle, because the photoemission process may introduce or modify spin polarization due to spin-orbit effects via the transition matrix element or other effects $[1,4]$. Spin polarization is the expectation value of the vectorial spin operator and defined as

$$
P_{\alpha}=\frac{I_{\alpha}^{\uparrow}-I_{\alpha}^{\downarrow}}{I_{\alpha}^{\uparrow}+I_{\alpha}^{\downarrow}} \quad \text { with } \alpha=(x, y, z)
$$

where $I_{\alpha}^{\uparrow, \downarrow}$ are measured photoelectron intensities with spin parallel or antiparallel to the cartesian axis $\alpha$. Photoemission intensities are determined by matrix elements, and the spin-orbit interaction may introduce spin dependent effects, thus affecting also the measured spin polarization. Fortunately, there are situations where these effects are absent or small, depending on the angular momentum character of the quantum state, the measurement geometry and the polarization of the exciting light. It is the merit of the Topical Review [1] to categorize the different matrix element and phase shift effects, illustrated by a large body of experimental work mainly from the Heinzmann group, and thereby serve as a valuable reference for future studies.

In condensed matter systems, delocalized valence band states can be spin polarized as a result of either the exchange interaction or the spin-orbit interaction. More than four decades ago Siegmann et al. [5] have shown that the spin-polarization of electrons photoemitted from a magnetic sample is related to its magnetization state. The 1980ies and early 90ies saw numerous studies related to the magnetism of surfaces and ultrathin films by spin-polarized photoemission by a small number of research groups. These activities later abated mainly because x-ray circular magnetic dichroism was introduced that 
provided more refined information on magnetic thin films, molecular adlayers and nanostructures, and with higher efficiency [6].

The last few years have seen a revival of interest in spin-polarized photoemission in the condensed matter physics community, as a unique method to study the dispersion of spin polarized surface states on non-magnetic solids [7]. Here, the spin-orbit interaction takes over the role of an external magnetic field or the exchange field. An ARPES study of a surface state on $\mathrm{Au}(111)$ by LaShell et al. [8] initiated this topic when they found an energy splitting proportional to the electron momentum, which they correctly interpreted as being due to the Rashba effect [9], which was hitherto known only in semiconductor physics. Experimental confirmation of the spin polarized and non-degenerate character came a few years later by Hoesch et al. [10] from spin-polarized ARPES (SARPES) data, which showed also the helical nature of the spin structure in these bands. The method has seen a veritable boost after the discovery of topological insulators [11,12] where SARPES data provided direct evidence for the existence of spinpolarized surface states, including their unconventional spin textures $[13,14]$.

In view of the considerable attention that these results find currently in the condensed matter physics community, and although the experiments have so far confirmed current theoretical expectations, it is important to critically assess how far the measurements of spin polarization vectors could potentially be affected by photoemission effects, and if not, to understand why. Light quanta do not interact directly with the spin degree of freedom, no problem there. But the dipole matrix element couples the orbital angular momentum of the initial state, i.e. the electron quantum state in the solid, to that of the final state, the photoelectron. The orbital angular momentum selection rules $\Delta \ell= \pm 1$ and $\Delta m=0, \pm 1$ result from this coupling. This is true to some degree also for delocalized valence states, because the matrix element can be written in the potential form where the spatial contributions are weighted by the gradient of the potential [15]. Photoemission is thus dominated by the core region where the quantum state can be described in terms of angular momentum $\ell$. In the presence of spin-orbit coupling the total angular momentum $j$ becomes relevant and the selection rule for the magnetic quantum number translates into $\Delta m_{j}=0, \pm 1$. This can lead to the emission of highly spin-polarized photoelectrons when spin-orbit effects are present either in the initial

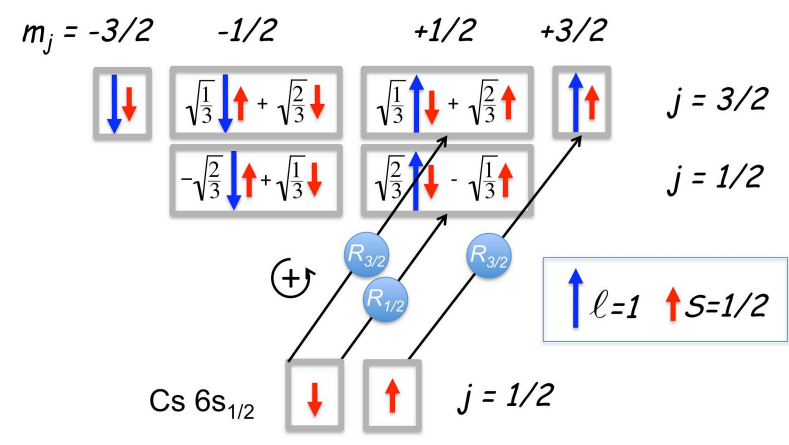

Fig. 1: Dipole allowed transitions from the $6 s 1 / 2$ level in a Cs atom excited by circularly polarized light into $p$ continuum states. In the final state the spin-orbit coupling produces states of total angular momentum $j=3 / 2$ and $j=1 / 2$ with corresponding radial transition matrix elements $R_{3 / 2}$ and $R_{1 / 2}$, respectively. For left-hand circularly polarized light we have $\Delta m_{j}=+1$.

or in the final state, or both. Note that the former case does not imply that the initial states are already spin polarized.

The orientation of the spin polarization vector is usually determined by symmetry considerations defining the relevant quantization axis in spin space, while the degree of spin polarization depends on dynamical effects and can depend strongly on the photon energy. The Fano-effect in atomic physics illustrates this nicely. Fig. 1 shows the dipole allowed transitions from a Cs $6 s$ orbital into a $p$ continuum state with spin-orbit coupling in the final state. Fano showed, by calculating the matrix elements in total angular momentum eigenstates and projecting them back onto the spin states, that the channel for producing photoelectrons with spin $s=-1 / 2$ can be completely closed by destructive interference when $R_{3 / 2}+2 R_{1 / 2}=0$ [16]. This condition is met at one particular energy at which a spin polarization of $100 \%$ is produced. The quantization axis is here the propagation direction of the circularly polarized light, i.e. the photon angular momentum direction, independent of the emission direction of the electrons.

Destructive interference in one spin channel can also occur due photoemission phase shifts between two continuum states with different orbital angular momenta resulting from the angular momentum selection rule [1]: for instance, emission out of a $p$ state produces continuum states of $s$ and $d$ character. Here, the orientation of the spin polarization vector can depend strongly on the emission angle relative to the photon angular momentum. By the same mechanism spin polarization can also be produced by linear polarized light, but only components that are 
perpendicular to the plane defined by the electric field vector of the light and the electron emission direction.

In solids these effects are complicated by the fact that spherical symmetry is broken and that different, competing quantization axes come into play, most prominently the direction normal to the surface, but also along dense atomic rows or along chiral axes. The overall consequence appears to be a general reduction of photoemission-induced spin polarization as compared to the atomic case. Nevertheless, very high values can be observed with circularly polarized light in heavy elements, where spin-orbit effects are strong, and where transitions between states with well defined total angular momenta can be resolved in the spectra. In these cases the photon angular momentum is the dominant quantization axis. When linear or unpolarized light is used on solids, spin polarization values produced by these effects do not exceed $10-20 \%$, and the quantization axis is given either by the light or by the crystal lattice [1].

The question posed in the title of this Viewpoint article cannot be answered conclusively at this point. It is clear that the matrix element and phase shift effects discussed in Ref. [1] do occur also in solids, especially in those containing heavy elements that are also of importance in Rashba systems and in topological insulators. As long as linear polarized light is used for excitation, one can safely say that the measured spin polarizations, which are typically very high, reflect mainly those of the electrons in the condensed matter system. Moreover, these 'intrinsic' spin polarizations can be expected to be stable with respect to variations in the photon energy and photon polarization direction, which may be used as a discriminating tool in the study of these systems. One example for this procedure is illustrated in Fig. 39 of Ref. [1], showing SARPES data from the topological insulator $\mathrm{PbBi}_{4} \mathrm{Te}_{7}$ measured at two different photon energies. The spin-polarization of the topological surface state remains high and approximately along the direction perpendicular to the in-plane momentum, while the states associated with the bulk conduction band show spurious polarization values.

There is still a lot of 'terra incognita' that spinpolarized photoemission experiments can explore in condensed matter systems. A recent example is provided by the unexpected finding of a strong Rashba-type spin-polarization in bulk states of bismuth [17]. It was explained by the internal reflection of Bloch states from the surface plane and the concomitant formation of standing electron waves in the direction perpendicular to the surface, in combination with the surface sensitivity of photoemission.

\section{Acknowledgements}

The author enjoyed many stimulating discussions with Ulrich Heinzmann, Hugo Dil and Thomas Greber.

\section{References}

[1] Heinzmann U and Dil J H 2012 J. Phys.: Condens. Matter X XXX

[2] Einstein A 1905 Ann. Phys. 17132

[3] Hüfner S 2003 Photoelectron Spectroscopy: Principles and Applications, $3^{\text {rd }}$ edn, Springer, Berlin Heidelberg New York

[4] Osterwalder J 2006 in Magnetism: A Synchrotron Radiation Approach, Lecture Notes in Physics Vol. 697, E. Beaurepaire, H. Bulou, F. Scheurer, J.-P. Kappler, eds, Springer Berlin Heidelberg New York, pp 95-120

[5] Busch G, Campagna M, Cotti P and Siegmann H C 1969 Phys. Rev. Lett. 22597

[6] Chen C T, Idzerda Y U, Lin H-J, Smith N V, Meigs G, Chaban E, Ho G H, Pellegrin E and Sette F 1995 Phys Rev. Lett. 75152

[7] Dil J H 2009 J. Phys.: Condens. Matter 21 403001

[8] LaShell S, McDougall B A, Jensen E 1996 Phys. Rev. Lett. 773419

[9] Bychkov Y A and Rashba E I 1984 J. Phys.: Condens. Matter 176039

[10] Hoesch M, Muntwiler M, Petrov V N, Hengsberger M, Patthey L, Shi M, Falub M, Greber T, Osterwalder J 2004 Phys. Rev. B 69 241401(R)

[11] Fu Liang, Kane C L and Mele E J 2007 Phys. Rev. Lett. 98106803

[12] Hasan M Z and Kane C L 2010 Rev. Mod. Phys. 823045

[13] Hsieh D, Xia Y, Wray L, Qian D, Pal A, Dil J H, Osterwalder J, Meier F, Bihlmayer G, Kane C L, Hor Y S, Cava R J, Hasan M Z 2009 Science 323 919

[14] Hsieh D, Xia Y, Qian D, Wray L, Dil J H, Meier F, Osterwalder J, Patthey L, Checkelsky J G, Ong N P, Fedorov A V, Lin H, Bansil A, Grauer D, Hor Y S, Cava R J, Hasan M Z 2009 Nature 4601101

[15] Jepsen D W 1979 Phys. Rev. B 20402

[16] Fano U 1969 Phys. Rev. 178131

[17] Kimura A, Krasovskii E E, Nishimura R, Miyamoto K, Kadono T, Kanomaru K, Chulkov E V, Bihlmayer G, Shimada K, Namatame H and Taniguchi M 2010 Phys. Rev. Lett. 105076804 